\title{
Mathematical model for calculating the force field of loads in the magnetic liquefied layer of electromechanical dispersants
}

\author{
Marina Bezzubceva, Vladimir Volkov* \\ Federal State Budgetary Educational Institution of Higher Education «Saint-Petersburg State Agrarian \\ University», Peterburgskoe shosse, 2, lit. A, Pushkin city, Saint Petersburg, 196601, Russia
}

\begin{abstract}
The article presents an analysis of the dominant dispersants in the production of the agro-industrial complex. A qualitative transition to the level of a priori reduction of energy costs for the most energy-intensive in the process flow and process instrumentation diagrams of the agro-industrial complex process of grinding of materials was found to be possible through the development and creation of devices, which, due to their design, increase the power of the force impact on the product to be processed. Numerous theoretical and practical studies within the framework of the scientific school "Efficient use of energy, intensification of technological processes" have confirmed that dispersants using electromagnetic fields, which form dispersing loads controlled by magnitude in energy-stressed contact interactions of ferro-bodies in their magnetic liquefied bed, can provide a product of a given quality with a minimum energy intensity indicator. The mathematical model of calculating the force field of loads in the magnetic liquefied bed of electromechanical dispersants presented in the article allows, as the practice has shown, developing the design schemes of electromagnetic mills, providing both the output of finished products with a high degree of selectivity, and with minimal energy consumption for the hardware arrangement of the technological process.
\end{abstract}

\section{Introduction}

Based on the analysis of mills, the possibility of intensification of the milling process in technological production lines has been established $[1,2,3,4]$ due to the introduction of electrophysical methods of forming dispersing loads in the magnetic liquefied bed of ferrobodies (milling elements of different configuration) [5]. As practice has shown, mills with the quasi-stationary alternating current magnetic field (vortex electromagnetic apparatuses VEA and electromagnetic mills - EMM [6,7] contribute to the intensification of the process due to the complex effect of high local pressures, cavitation, friction, and agitation on the material throughout the volume of the working chamber for material processing. Meanwhile, in the devices of this group, the formation of dispersing loads is due to the chaotic physical and mechanical processes in the layer of grinding elements, which complicates the introduction into the production technology of effective management of grinding quality with

\footnotetext{
* Corresponding author: vol9795@yandex.ru
} 
obtaining the selectivity indicators specified by the standards. Effective management of force and energy influences on the particles of the processed product is possible by introducing a magnetic liquefied bed of ferromagnetic grinding bodies of different structural modifications into the design of the grinder.

\section{Materials and Methods}

The method of high-speed cinematography was used in the research. The theoretical studies are based on Maxwell's electrophysical models.

\section{Results}

Currently, electromechanical dispersants (EMD) have been developed and tested in production practice to provide reliable control of selectivity parameters of the grinding process by regulating the frequency and force of impact on the particles of the processed product in the magnetic liquefied bed of grinding elements (ferro-bodies) [1]. The fields of application of EMDs are shown in Fig. 1.

The studies are based on Maxwell's fundamental theory "on the dipole interaction of ferroballs in a magnetic field during the formation of force-contact interactions" [8,9].

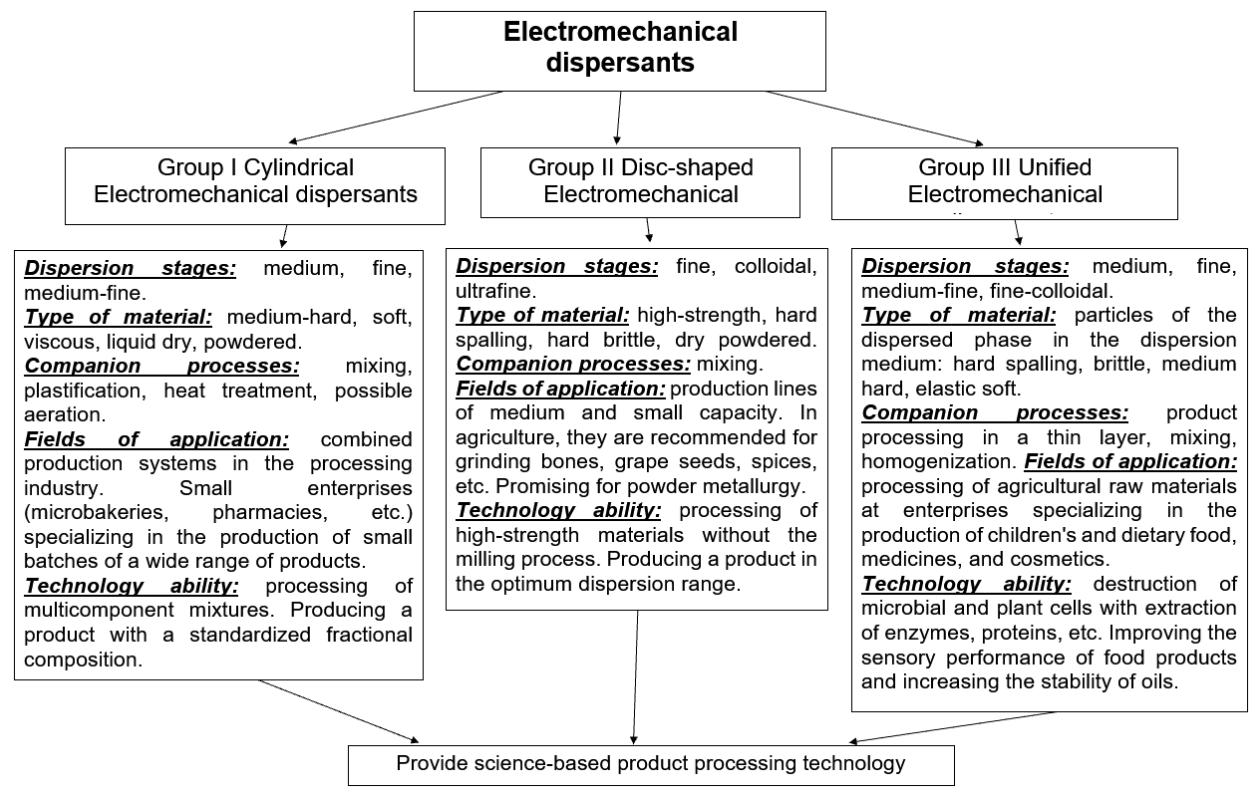

Fig. 1. Technological purpose of electromechanical dispersants.

Design schemes of EMD represent the subject of inventions. The objects of intellectual property of the scientific school "Efficient use of energy, intensification of electrotechnological processes" on the subject of electromechanical activation of the process of grinding materials are presented in Fig. 2. 


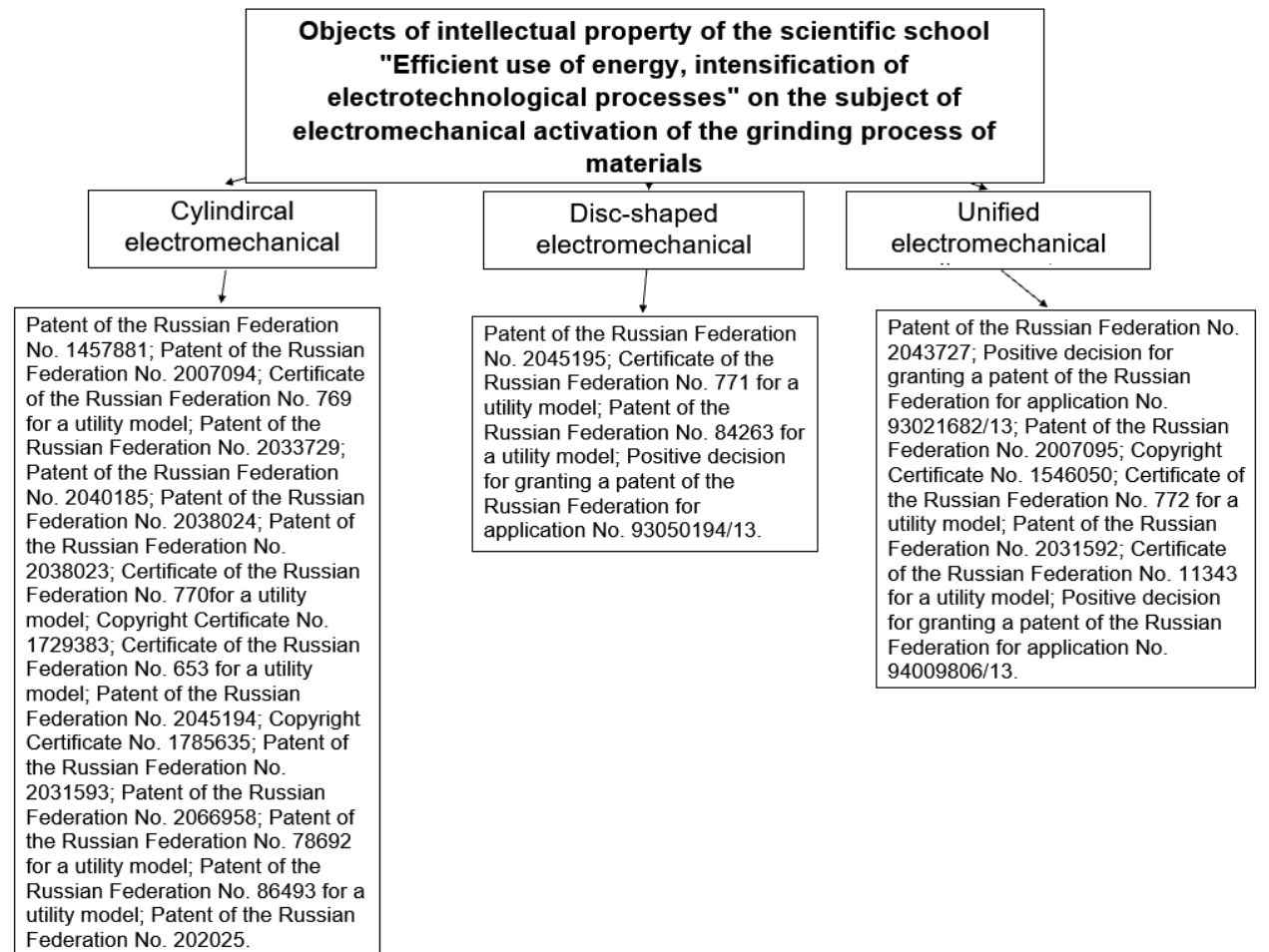

Fig. 2. Objects of intellectual property of the scientific school "Efficient use of energy, intensification of electrotechnological processes" on the subject of electromechanical activation of the grinding process of materials.

The formation cluster of dispersing loads (shock, compression, and abrasion) [10] in a ferrodynamic medium of spherical milling bodies is shown in Fig. 3a, b, c, and d.

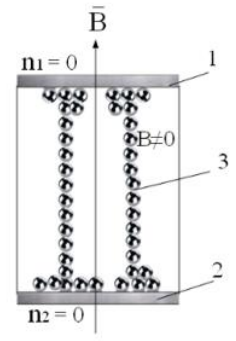

a

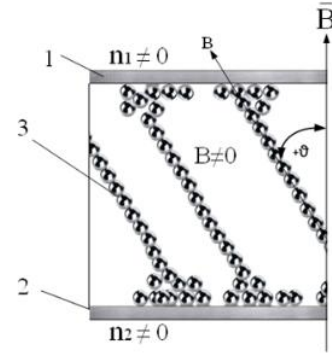

b

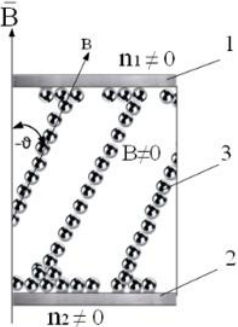

C

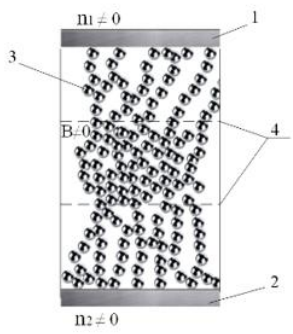

d

Fig. 3. Formation cluster of dispersing loads in the ferrodynamic medium of the EMD working volume: 1,2 - surfaces limiting the grinding chamber; 3 - ferroballs (elements of the magnetic liquefied bed); 4 - the boundary of the ferroball reorientation layer; B - vector of electromagnetic field induction; $\mathrm{n}$ - offset frequency (or rotation if coaxial cylinder surfaces are used) of surfaces (external and internal respectively) of the working chamber; $\mathrm{v}$ - deformation angle of the chain of ferro-bodies: $\mathrm{a}$ - intermediate structure of formation of compressive loads in the ferrodynamic medium (at $n=0)$; $\mathrm{b}, \mathrm{c}-$ the intermediate structure of formation of compressive and abrasive loads in the ferrodynamic medium; $\mathrm{d}$ - the structure of formation of shock-compressive and abrasive loads in the ferrodynamic medium.

The mathematical modeling of force contacts in a ferrodynamic medium is based on the development of Maxwell's physical model of the interaction of metal balls in a magnetic field [3]. Given the Maxwell dipole model, the force $\bar{F}=\left(F_{x}, F_{y}, F_{z}\right)$ is defined by the expressions [4] 


$$
\left.\begin{array}{l}
F_{X}=\frac{1}{2} \frac{\mu-1}{\mu+2} R_{0}^{3} \frac{\partial H^{2}}{\partial x} ; \\
F_{y}=\frac{1}{2} \frac{\mu-1}{\mu+2} R_{0}^{3} \frac{\partial H^{2}}{\partial y} ; \\
F_{Z}=\frac{1}{2} \frac{\mu-1}{\mu+2} R_{0}^{3} \frac{\partial H^{2}}{\partial z} .
\end{array}\right\}
$$

where $\mu$-magnetic permeability; $H$ - electromagnetic field strength.

The interaction force in the contact system of two ferroballs with radius $R_{0}$ in the considered ferrodynamic medium

$$
F r=\left.\frac{1}{2} \frac{\mu-1}{\mu+2} R_{0}^{3} \frac{\partial H^{2}}{\partial r}\right|_{r=2 R_{0}}
$$

Considering that the scalar magnetic potential describing the field outside the sphere in a homogeneous magnetic field is defined by the formula

$$
\Phi e=H_{0}\left(r-\frac{\mu-1}{\mu+2} \frac{R_{0}^{3}}{r^{2}}\right) \cos v
$$

and at a point with spherical coordinates $\mathrm{r}, \mathrm{v}, \varphi$, the magnetic field strength induced by this potential is as follows:

$$
\left.\begin{array}{c}
H r=\frac{\partial \Phi e}{\partial r}=H_{0}\left(1+2 \frac{\mu-1}{\mu+2} \frac{R_{0}^{3}}{r^{3}}\right) \cos v \\
H v=\frac{1}{r} \frac{\partial \Phi e}{\partial v}=-H_{0}\left(1-\frac{\mu-1}{\mu+2} \frac{R_{0}^{3}}{r^{3}}\right) \sin v \\
H \varphi=0
\end{array}\right\}
$$

then the interaction force of the ferroballs (or the force of the ferrodynamic medium on the processed product) can be defined by the formula

$$
F r=\left.\frac{1}{2} \frac{\mu-1}{\mu+2} R_{0}^{3} \frac{\partial H^{2}}{\partial r}\right|_{r=2 R_{0}}
$$

Or

$$
F r=-\frac{3}{256} H_{0}^{2} R_{0}^{2} \frac{(\mu-1)^{2}}{(\mu+2)^{3}}[(13 \mu+11)+9(3 \mu+5) \cos 2 v]
$$

The momentum of the interaction forces of the field with the elements of the ferrodynamic medium has the form

$$
M_{U}=-\frac{3}{128} H_{0}^{2} R_{0}^{2} \frac{(\mu-1)^{2}}{(\mu+2)^{3}}(17 \mu+31) \sin 2 v
$$

where $v$-deformation angle of the structural group of ferroballs.

The change in the sign in front of formulas 5 and 6 indicates a change in the attraction force between the ferroballs by the repulsion force. This explains the dynamism of the formation of the impact and abrasion forces between the ferroballs in their magnetical liquefied bed. It should be noted that the contact interactions of the elements take place through the interlayer of the material to be milled. Knowing the strength characteristics of the processed material, it is possible to set the energy and force parameters specified by the technology in the working volume of electromechanical dispersants. Such an approach a priori eliminates energy losses and contributes not only to reducing the energy intensity of the output product but also to improving the quality due to the alignment of the particle size distribution and increasing the selectivity of the process. 
A qualitative representation of the formation and destruction of structural groups of milling ferromagnetic elements with the formation of shock and abrasion loads on the particles of the product to be processed when the sign in formula 5 is changed is shown in Fig. 4.

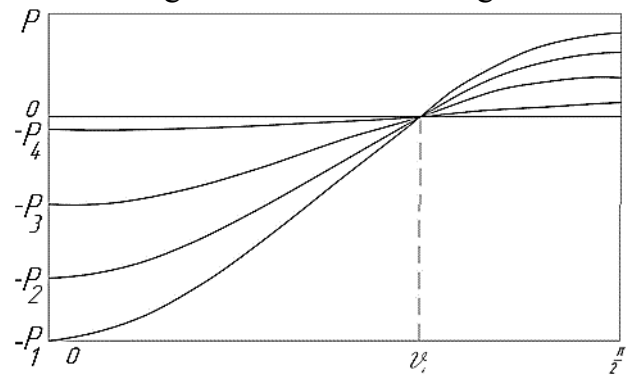

Fig. 4. Qualitative representation of the formation and destruction of structural groups of milling ferromagnetic elements.

\section{Conclusion}

Based on the conducted research, a mathematical model for calculating the forces and momenta acting on the ferrodynamic medium in the working volumes of electromagnetic mills has been developed. It has been established that the value of the deformation angle of the chains of ferroballs in the formation cluster of dispersing loads in the volumes of working chambers of dispersants determines the force of interaction between these elements in their magnetic liquefied bed and is a parameter characterizing the dynamics of the material grinding process. As practice has shown, the development of EMD design schemes based on calculations of the force field of loads according to the presented mathematical model allows producing finished products with a high degree of selectivity that meets the requirements of standards.

\section{References}

1. Aldona Kluczek, International Journal of Production Economics, 216, 190-203, (2019) https://doi.org/10.1016/j.ijpe.2019.04.016.

2. Gökan May, Ilaria Barletta, Bojan Stahl, Marco Taisch, Applied Energy, 149, 46-61, (2015) https://doi.org/10.1016/j.apenergy.2015.03.065.

3. A.S. Yanyushkin D.A. Rychkov D.V. Lobanov, Procedia Engineering, 150, 942-947, (2016) https://doi.org/10.1016/j.proeng.2016.07.067.

4. Stefanie Hackenberg, Mario Jekle, Food Chemistry, 248, 296-303, (2018) https://doi.org/10.1016/j. foodchem.2017.12.054.

5. Bezzubceva M.M., Volkov V.S. Applied research of electromagnetic mechanical activators: monograph, Saarbrucken Lambert Academic Publishing (2016)

6. Bezzubceva M.M., Volkov V.S. Mechanical activators of the agro-industrial complex. Analysis, innovations, inventions: monograph, SPb. (2014)

7. Bezzubceva M.M., Volkov V.S., KrishtopaN.Yu., Mastepanenko M.A., Gabrielyan Sh. Zh. Theoretical Studies of the Method of Electromagnetic Mechanical Activation, The Challenge of Sustainability in Agricultural Systems, Berlin: Springer (2021)

8. Misbah Ijaz, Muhammad Ayub, Heliyon, 5, 4, 01465. (2019) https://doi.org/10.1016/j.heliyon.2019.e01465.

9. Tweney, R.D., Science \& Education, 20 (7-8), 687-700 (2011)

10. Hossein Ahmadian A, B, Mojtaba Ghadiri Advanced Powder Technology, 32 (1), 204210 (2021) https://doi.org/10.1016/j.apt.2020.12.001. 